\title{
Acoustic Radiation Force Impulse Imaging
}

National Cancer Institute

\section{Source}

National Cancer Institute. Acoustic Radiation Force Impulse Imaging. NCI Thesaurus.

Code C116484.

A type of elastography that uses acoustic radiation force to generate images of the mechanical properties of soft tissue. 